\title{
Tuning electrical properties of hierarchically assembled Al-doped ZnO nanoforests by room temperature Pulsed Laser Deposition
}

P Gondoni ${ }^{1}$, P Mazzolini ${ }^{1,2}, \mathrm{~V}$ Russo $^{1}, \mathrm{M} \mathrm{Diani}^{1}, \mathrm{M} \mathrm{Amati}^{3}, \mathrm{~L}_{\text {Gregoratti }}{ }^{3}, \mathrm{~V}$ De Renzi ${ }^{4,5}$, G C Gazzadi ${ }^{5}$, J Martí-Rujas², A Li Bassi ${ }^{1,2}$ and C S Casari ${ }^{1,2}$

${ }^{1}$ Dipartimento di Energia and NEMAS Center for NanoEngineered Materials and Surfaces, Politecnico di Milano, via Ponzio 34/3, 20133 Milano (Italy)

2 Center for Nano Science and Technology@Polimi, Istituto Italiano di Tecnologia, Via Pascoli 70/3, 20133 Milano (Italy)

3 Elettra - Sincrotrone Trieste S.C.p.A., s.s. $14 \mathrm{~km} 163.5$ in Area Science Park, 34149 Trieste (Italy)

4 Universita' di Modena e Reggio Emilia,

Dipartimento di Scienze Fisiche Informatiche e Matematiche, via G. Campi 213/a, (Italy) and ${ }^{5}$ CNR-Institute of Nanoscience-S3, Via G. Campi 213/a, 41100 Modena (Italy)

Large surface area, 3D structured transparent electrodes with effective light management capability may represent a key component in the development of new generation optoelectronic and energy harvesting devices. We present an approach to obtain forest-like nanoporous/hierarchical Al-doped $\mathrm{ZnO}$ conducting layers with tunable transparency and light scattering properties, by means of room temperature Pulsed Laser Deposition in a mixed Ar: $\mathrm{O}_{2}$ atmosphere. The composition of the background atmosphere during deposition can be varied to modify stoichiometry-related defects, and therefore achieve control of electrical and optical properties, while the total background pressure controls the material morphology at the nano- and mesoscale and thus the light scattering properties. This approach allows to tune electrical resistivity over a very wide range $\left(10^{-1}-10^{6} \Omega \mathrm{cm}\right)$, both in the in-plane and cross-plane directions. Optical transparency and haze can also be tuned by varying the stoichiometry and thickness of the nano-forests. 


\section{INTRODUCTION}

Aluminum doped Zinc Oxide (AZO) is an inexpensive and performing transparent conducting material employed in optoelectronic and energy conversion devices as a replacement for Indium-Tin Oxide, which is widely used yet scarce and expensive [1-3]. For a suitable application in latest photovoltaic devices (e.g. organic or hybrid next generation solar cells) AZO must meet novel requirements which are not limited to optical transparency and electrical conductivity, but include light scattering, large surface area and compatibility with flexible polymer substrates (i.e. no deposition or post-deposition treatments at high temperature) [4-7].

Conventional polycrystalline AZO films to be employed as Transparent Conducting Oxide (TCO) layers can be grown using different techniques (e.g. sputtering, Pulsed Laser Deposition), even at room temperature, [8-14] and are typically characterized by low electrical resistivities, of the order of $10^{-4} \Omega \mathrm{cm}$, and an average optical transparency of the order of $85-90 \%$, but cannot provide effective light management in terms of light diffusion and trapping.

Conversely, mesoporous/hierarchical structures can be optimized for maximizing light scattering and diffusion and we have recently demonstrated the synthesis of hierarchically nanostructured AZO layers resembling a nanotree forest [14] by room temperature Pulsed Laser Deposition (PLD), performed in the presence of $\mathrm{O}_{2}$ as a background gas at relatively high pressures, i.e. $>10 \mathrm{~Pa}$. These structures are characterized by optical transmittance above $85 \%$, with a haze (i.e. scattered to transmitted light intensity ratio) over $80 \%$ and large specific surface area; however, they are also characterized by extremely high in-plane electrical resistivity (of the order of $\mathrm{M} \Omega \mathrm{cm}$ ) [14, 15]. On the contrary, PLD at a lower oxygen pressure can be used to deposit conventional, 'compact' polycrystalline AZO layers with state-of-the-art electrical conductivity.

The different properties of the two types of structures ('compact' vs 'nanoporous') are due both to their different morphology, but also, in the case of PLD synthesis, to their chemical composition (i.e. oxygen content). In particular, for high oxygen pressure, collisions between the ablated species and the background gas molecules cause the nucleation of clusters which, reaching the substrate with low kinetic energy [16], give rise to porous, hierarchically assembled structures resembling a nanoforest [14]. Unlike compact layers ob- 
tained at low pressures, these porous films are not uniformly connected due to their open morphology, which is detrimental to electrical conduction [15]. Moreover, the influence of oxygen pressure on the properties of the material is not limited to morphology, since films grown at different $\mathrm{O}_{2}$ pressures are also different in chemical composition. Although the role and the importance of stoichiometry and defects in $\mathrm{ZnO}$-based materials (e.g. O vacancies, $\mathrm{O}$ segregation at grain boundaries, complex interplay between $\mathrm{O}$ vacancies and extrinsic dopant atoms) are not trivial and have long been discussed with different interpretations [17-20] both in terms of electrical and optical properties, it is established that deposition in O-rich environment is typically detrimental for the electrical transport properties and in particular for charge carrier concentration.

In this work we develop an approach to uncouple the two above mentioned effects of the deposition atmposphere, in order to improve the electrical conductivity of strongly light scattering AZO hierarchical porous nanostructures, by combining a low oxygen content which should be beneficial for electrical properties [21] with an open morphology optimized for light diffusion [14] and surface area. This approach is based on performing PLD in a mixed background atmosphere, constituted by an inert gas (Ar) and oxygen. In particular, the total deposition pressure controls morphology, while variations in the $\mathrm{O}_{2}$ partial pressure directly influence oxygen content and stoichiometry of the material. We show how, by maintaining the total deposition pressure constant and varying the partial oxygen pressure, it is possible to obtain multifunctional structures, i.e. transparent and conducting hierarchically assembled forest-like films, with a gain in conductivity up to 6 orders of magnitude (with respect to deposition in pure $\mathrm{O}_{2}$ ) and significant light scattering properties.

\section{EXPERIMENTAL}

AZO thin films were deposited on soda-lime glass and $\mathrm{Si}(100)$ substrates by PLD at room temperature. A solid (2\%wt.) $\mathrm{Al}_{2} \mathrm{O}_{3}: \mathrm{ZnO}$ target (corresponding to a $3.2 \%$ at. Al content) was ablated by a pulsed UV laser (Nd:YAG 4th harmonic, $\lambda=266 \mathrm{~nm}$, pulse duration $\approx 6 \mathrm{~ns}$, pulse energy $75 \mathrm{~mJ}$, repetition rate $10 \mathrm{~Hz}$ ). The target-to-substrate distance was fixed at $50 \mathrm{~mm}$, with an energy density of about $1 \mathrm{~J} \mathrm{~cm}^{-2}$. The background atmosphere was obtained by different combinations of $\mathrm{Ar}$ and $\mathrm{O}_{2}$, flowing in the deposition chamber through mks 2179a mass flow controllers, with a total pressure in the chamber of 100-110 
$\mathrm{Pa}$ and an $\mathrm{O}_{2}$ content ranging from $0 \%$ to $100 \%$. For most of the investigated samples deposition time was fixed at about 180 minutes (108000 laser shots) corresponding to a nominal thickness of about $2 \mu \mathrm{m}$; the deposition time was adjusted for deposition of thinner (about $300 \mathrm{~nm}$ ) and thicker films when necessary, as discussed in the Results section. Postdeposition thermal treatments $\left(500^{\circ} \mathrm{C}\right.$, annealing time 1 hour $)$ in vacuum $\left(p<5 \cdot 10^{-5} \mathrm{~Pa}\right)$ or in air at atmospheric pressure were performed for some of the samples in a homemade furnace and in a Lenton muffle furnace, respectively.

In-plane electrical properties were measured in the 2-point (for resistivities of $100 \Omega \mathrm{cm}$ and higher) and 4-point probe (for resistivities up to $100 \Omega \mathrm{cm}$ ) configurations with a Keithley K2400 Source/Measure Unit as a current generator (from $100 \mathrm{nA}$ to $10 \mathrm{~mA}$ ), an Agilent 34970A voltage meter and a 0.57 T Ecopia permanent magnet. Due to difficulties in applying contacts onto the porous surface without damaging the film, the current was injected from the bottom (i.e. at the substrate/film interface) through evaporated Au contacts.

For cross-plane electrical measurements, AZO layers were deposited on commercially available conducting ITO substrates $\left(\mathrm{R}_{S}=10 \Omega / \square\right)$. The measurements were performed inside a Dual Beam (FIB-SEM) system using a two-probe setup: one electrode was fixed on the ITO substrate by silver paste, the second electrode was a tungsten tip mounted on a piezo nanomanipulator that could be moved around on the AZO film. The electrodes were connected to a Keithley sourcemeter supplying the bias voltage and measuring the current down to a sensitivity of $0.01 \mathrm{pA}$. On each sample, as a first check, an I-V curve was measured on the ITO substrate, always obtaining the expected low-resistive ohmic behaviour in agreement with the nominal sheet resistance value of $10 \Omega / \square$.

Optical transmittance, haze and absorption spectra were acquired with a UV-vis-NIR PerkinElmer Lambda 1050 spectrophotometer with a $150 \mathrm{~mm}$ diameter integrating sphere. Scanning Electron Microscope Images were acquired with a Zeiss SUPRA 40 field emission SEM. X-Ray Diffraction was performed in $\theta-2 \theta$ configuration (Bruker D8 Advance Diffractometer $\mathrm{Cu} \mathrm{K} \mathrm{K}_{\alpha 1}, \lambda=1.5406 \AA$ ). Relative variations in oxygen content were estimated by spatially resolved X-ray photoemission spectroscopy performed at the ESCA microscopy beamline of the Elettra Synchrotron facility in Trieste, Italy [22, 23]. The X-ray beam is focused on the cross-section of the sample to a diameter of about $130 \mathrm{~nm}$ using Fresnel zone plate optics, and the sample can be raster scanned with respect to the microprobe. Photoemission spectra were measured with a $650 \mathrm{eV}$ photon energy with $0.2 \mathrm{eV}$ energy resolution. 


\section{RESULTS AND DISCUSSION}

\section{A. Morphology and structural properties}

Hierarchically assembled AZO layers were obtained at a total deposition pressure of 110 $\mathrm{Pa}$; the pressure range was chosen relying on previous works [14] where oxygen background pressure and gas flow rate were studied as parameters to obtain hierarchically structured morphology, with porosity at the mesoscale. Cross-sectional SEM images of the materials grown at $110 \mathrm{~Pa}$ with different oxygen content are shown in Fig.1.
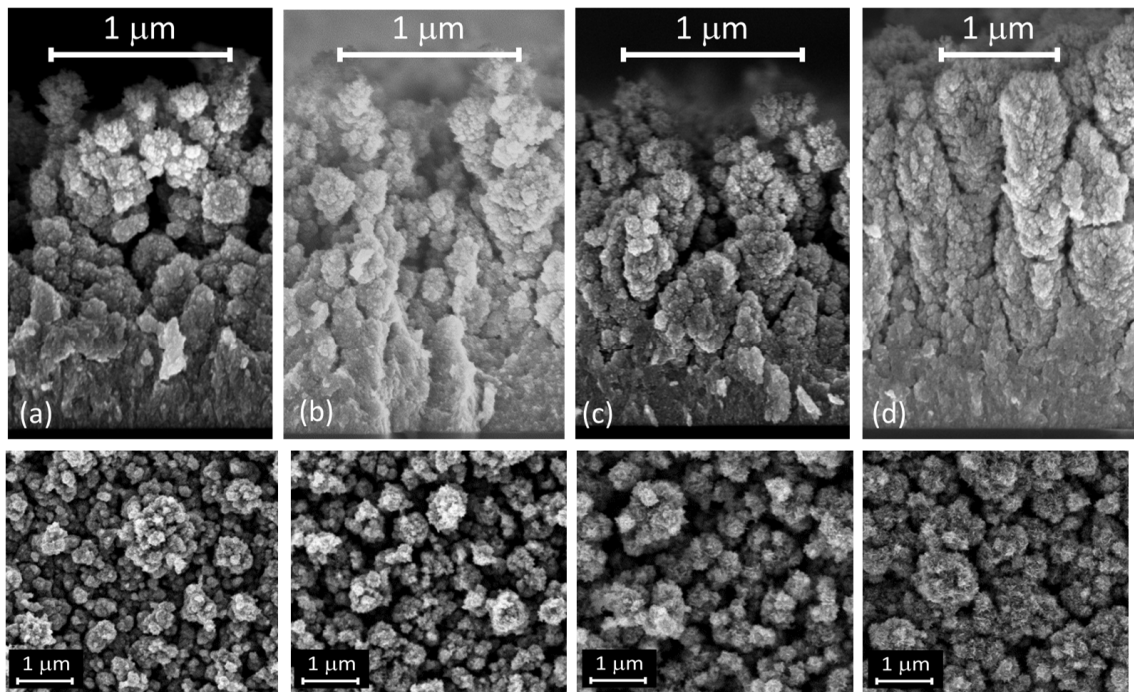

FIG. 1: Top line: cross sectional SEM images of hierarchical AZO layers grown at a total deposition pressure of $110 \mathrm{~Pa}$, with $2.5 \% \mathrm{O}_{2}$ (a), 6\% $\mathrm{O}_{2}$ (b), $10 \% \mathrm{O}_{2}$ (c) and $100 \% \mathrm{O}_{2}(\mathrm{~d})$. Bottom line: corresponding top view SEM images.

For the layers shown in Fig.1 (nominal thickness $2 \mu \mathrm{m}$ ) the inert part of the atmosphere was constituted by argon, and the $\mathrm{O}_{2}$ content was 2.5\% (Fig.1a), 6\% (Fig.1b), 10\% (Fig.1c) and 100\% (pure oxygen, Fig.1d) respectively. Helium was also tested as an inert gas, however depositions performed in $\mathrm{He}: \mathrm{O}_{2}$ atmosphere in a similar pressure range did not generate hierarchical structures; in fact it is known that usually a much higher 
He pressure is needed to grow nanoporous oxide structures [24], due to the small mass of He atoms, whose collisions with the ablated species from the target do not allow sufficient clustering phenomena and kinetic energy decrease, as observed by Geohegan et al. [25].

The images reported in Fig.1 show the change from a more open and disordered porous morphology towards the gradual arise of a preferential growth direction orthogonal to the substrate surface, enhancing the hierarchical nature of the material which resembles a nanotree forest in the case of pure oxygen (Fig.1d). Regardless of the deposition parameters, the bottom layer of the film is more compact and uniformly connected; then the structure of the material evolves moving away from the substrate, becoming more open and porous. In the film top region the influence of the background deposition atmosphere is more significant, and the forest-like morphology clearly appears at high oxygen content. The top view SEM morphology at the mesoscale does not significantly differ for different deposition atmospheres.

Structural characterization performed by X-Ray Diffraction (XRD) reveals a nanocrystalline structure for all the AZO layers. The most intense reflections of $\mathrm{ZnO}$ powders appear in the diffractograms, as it can be observed in Fig.2; however the peak intensity ratio suggests a slightly preferential orientation along the (002) direction (as already observed in a more marked way for conventional 'compact' AZO films [21]). No Zn XRD reflections that may be related to $\mathrm{Zn}$ clustering were observed. The mean size of the crystal domains was calculated from the width of the (002) peak using Scherrer formula, and the values are reported in Fig.2b. The calculated domain size is of the order of $20 \mathrm{~nm}$ for all oxygen partial pressures, except for the case of pure argon (oxygen-free atmosphere), with a mean domain size of about $14 \mathrm{~nm}$; this can be interpreted as an indication of the presence of a larger amount of structural defects (e.g. oxygen vacancies). As anticipated above, the importance of such defects in $\mathrm{ZnO}$-based materials has widely been discussed, and in our previous works $[14,21]$ we identified a significant correlation between electrical properties and pure oxygen deposition pressure of compact AZO layers grown by PLD at room temperature. Calculations of the $c$ lattice parameter from Bragg's formula were also performed, indicating only small variations from the $\mathrm{ZnO}$ reference value of $5.20 \AA[2]$. 

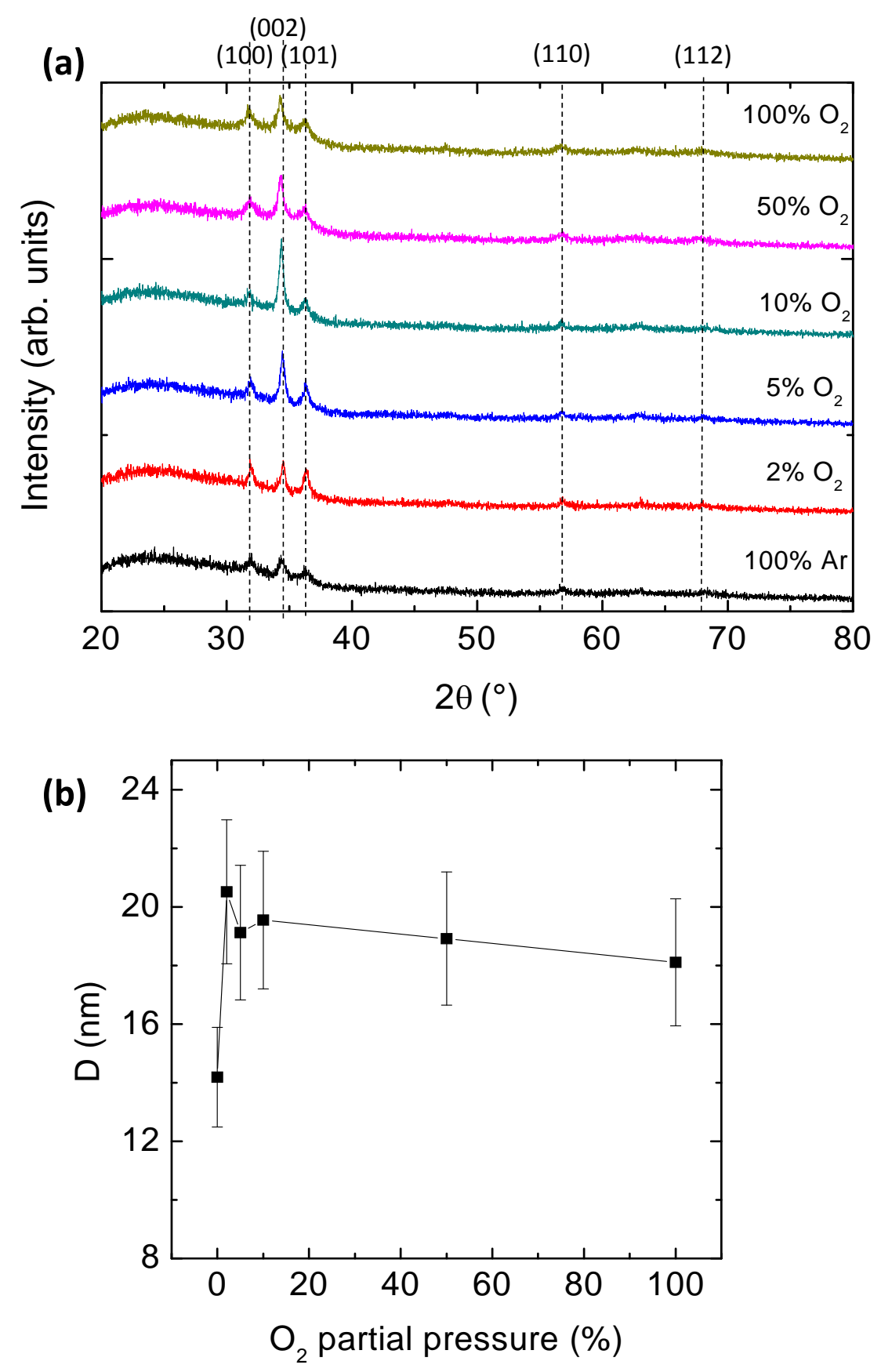

FIG. 2: (a) XRD patterns of porous AZO layers (300 nm thick) grown at $100 \mathrm{~Pa}$ with different oxygen partial pressures. The most intense reflections of $\mathrm{ZnO}$ powders are present, with hints of a preferential orientation in the (002) direction. (b) Mean domain size $D$, calculated from Scherrer's formula on the (002) peak, as a function of oxygen partial pressure. 


\section{B. Chemical composition and stoichiometry}

The effects of oxygen partial pressure during deposition on the chemical composition of AZO layers were qualitatively evaluated by performing cross-sectional X-ray Photoemission Spectroscopy (XPS), with $\mathrm{a} \approx 130 \mathrm{~nm}$ focused beam of synchrotron X-ray radiation as an excitation source, on selected samples. Measurements were performed on layers grown at a total pressure of $110 \mathrm{~Pa}$, both in pure $\mathrm{O}_{2}$ and in a $6 \% \mathrm{O}_{2}$ atmosphere. In particular, variations in the $\mathrm{Zn} / \mathrm{O}$ stoichiometry ratio between the samples were estimated by acquiring single point XPS spectra in different regions of the nanoforest cross-section of very thick films (thickness $>10 \mu \mathrm{m}$ ), in order to check composition uniformity within each sample.

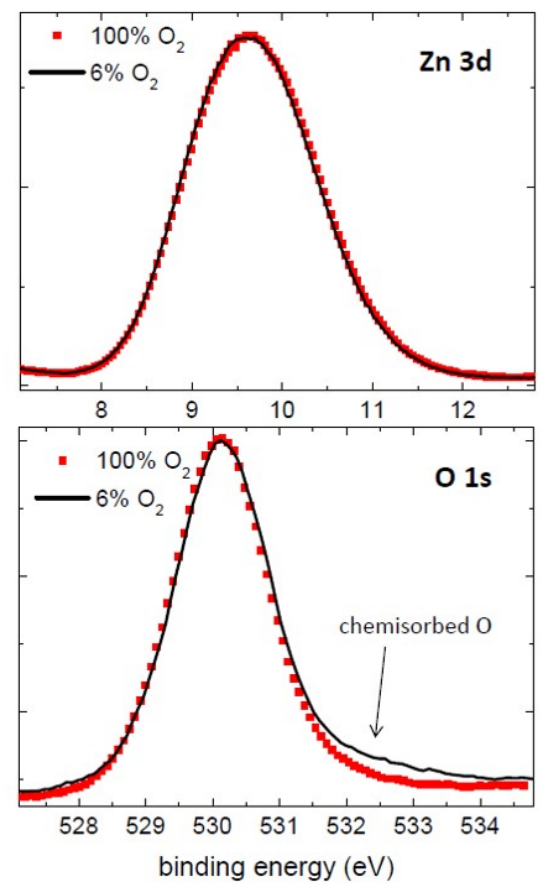

FIG. 3: XPS Zn 3d (top) and O 1s (bottom) peaks for the AZO layers deposited at $6 \%$ and $100 \%$ oxygen content.

Changes in the $\mathrm{O} / \mathrm{Zn}$ stoichiometry ratio $\left(n_{O} / n_{Z n}\right)$ between the investigated samples were estimated by comparing the ratio of the integral intensities of the $\mathrm{O} 1 \mathrm{~s}$ and $\mathrm{Zn} 3 \mathrm{~d}$ or $\mathrm{Zn} 3 \mathrm{p}$ photoemission lines, averaged over different points of the cross-section surface, in AZO samples grown in different conditions; this ratio is proportional to $n_{O} / n_{Z n}$ through the photoionization cross-section and the electron escape depth. The $\mathrm{O} / \mathrm{Zn}$ intensity ratio for AZO grown at low (6\%) oxygen partial pressure was about $80 \%$ of the $\mathrm{O} / \mathrm{Zn}$ ratio of 
nominally 'stoichiometric' $\left(100 \% \mathrm{O}_{2}\right)$ AZO. This significant variation allows to safely state that the oxygen partial pressure during deposition directly influences the oxygen content and the stoichiometry of the material. An analysis of the lineshape of $\mathrm{Zn} 3 \mathrm{~d}$ peaks (see Fig.3) did not reveal significant differences or chemical shifts between the two samples. Only in the $\mathrm{O} 1 \mathrm{~s}$ peak of the sample deposited at $6 \%$ oxygen partial pressure a very small component at about $532 \mathrm{eV}$, in addition to the main feature at $530 \mathrm{eV}$, indicates the presence of a small amount of chemisorbed oxygen [26]. We observe that a quantitative evaluation of $\mathrm{Zn}$ oxidation state is complicated by the complex, composite structure of our samples, comprising nanocrystalline domains and a large fraction of (disordered) grain boundaries and surface, due to the porous, nanoparticle-assembled morphology, and also considering that XPS is sensitive only to the surface (few $\mathrm{nm}$ ) of the nanoparticles.

\section{Optical properties}

The investigation of optical transparency and light scattering was carried out by measuring the total and diffuse transmittance for the AZO nanoforest layers. The corresponding attenuation coefficient $\alpha$ and haze factor were calculated; the $\alpha$ average value in the visible $(400 \mathrm{~nm}-700 \mathrm{~nm})$ range is reported in Fig.4.

The values of the attenuation coefficient show a significant decrease as the oxygen partial pressure is increased; AZO grown in pure Ar is strongly absorbing in the visible range $\left(\alpha \approx 2.8 \cdot 10^{4} \mathrm{~cm}^{-1}\right.$ ) and then the attenuation coefficient decreases by over one order of magnitude as the oxygen content in the deposition chamber (hence in the film) increases,

down to $1400 \mathrm{~cm}^{-1}$ for a pure $\mathrm{O}_{2}$ deposition atmosphere. The calculated values of $\alpha$ are compatible also for layers of different thickness. This corresponds to a mean trasnmittance of the order of $60 \%$ for $2 \mu \mathrm{m}$ thick layers and of $90 \%$ for $300 \mathrm{~nm}$ thick layers, except for the case of deposition in pure Ar, for which thick layers are opaque to visible light while $T \approx 55 \%$ for $300 \mathrm{~nm}$ layers. This trend in optical transparency could be readily explained by considering the effects of $\mathrm{O}$ vacancies on the available electron states of doped and undoped $\mathrm{ZnO}$ [27], and a similar behaviour was found in our previous works [14, 21] for AZO grown by PLD in pure $\mathrm{O}_{2}$ at different deposition pressures; oxygen deficiency in AZO creates available defect states within the energy gap, which allow for optical transitions at energy lower than the optical gap, i.e. in the visible range. 


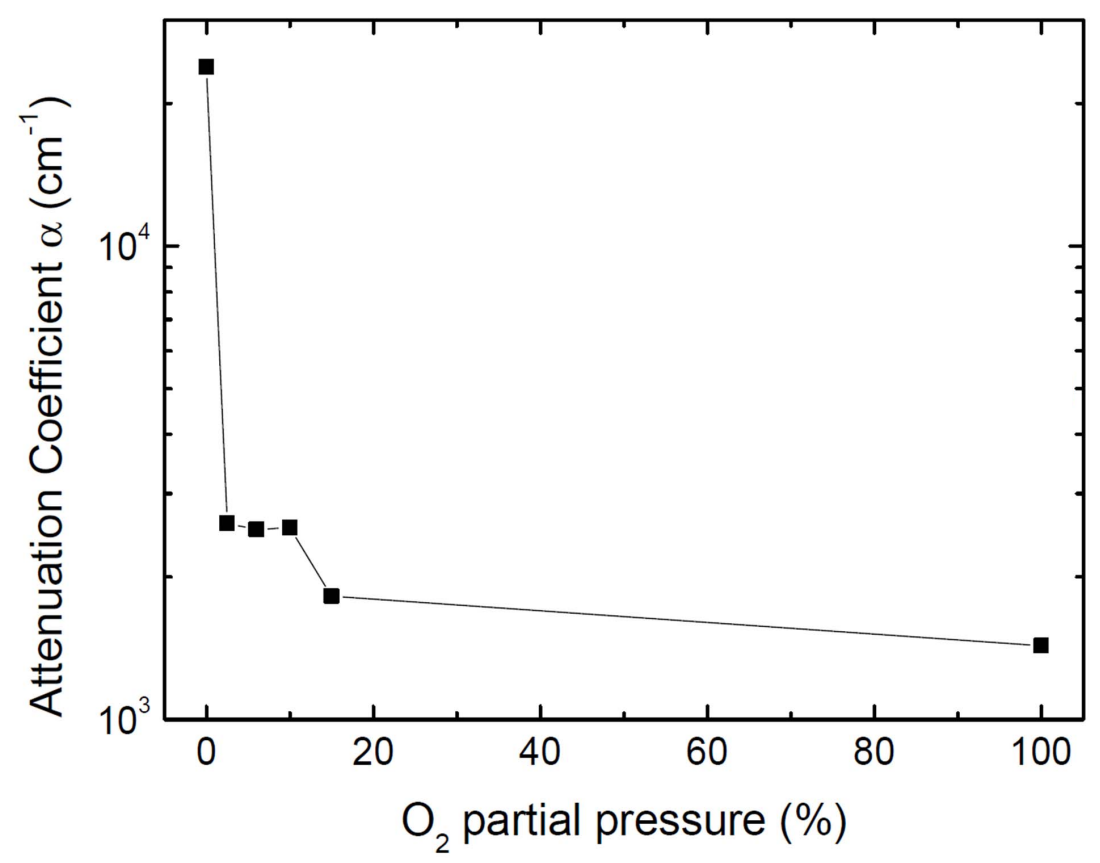

FIG. 4: Attenuation coefficient of AZO mesoporous layers as a function of $\mathrm{O}_{2}$ deposition partial pressure.

The average haze in the visible range $(400 \mathrm{~nm}-700 \mathrm{~nm}$ ) is larger than $95 \%$ for all deposition conditions in the case of $2 \mu \mathrm{m}$ thick films, witnessing the strong light scattering potential of the mesoporous morphology; this means that virtually all the transmitted photons are scattered by the hierarchical structures. Haze is quite high also in the case of thinner films (for instance it is about $40 \%$ for a $300 \mathrm{~nm}$ thick layer grown at $6 \%$ oxygen content). This means that it is possible to achieve control of light scattering in mesoporous AZO by varying either the oxygen partial pressure or the film thickness, and it is possible to know how both parameters influence total transparency (transmittance) by using Lambert-Beer's law on the values in fig. 4 .

\section{Electrical transport properties}

The measured in-plane electrical resistivity of hierarchical AZO layers is reported in Fig.5 as a function of $\mathrm{O}_{2}$ partial pressure during deposition (as measured for $2 \mu \mathrm{m}$ thick films). 


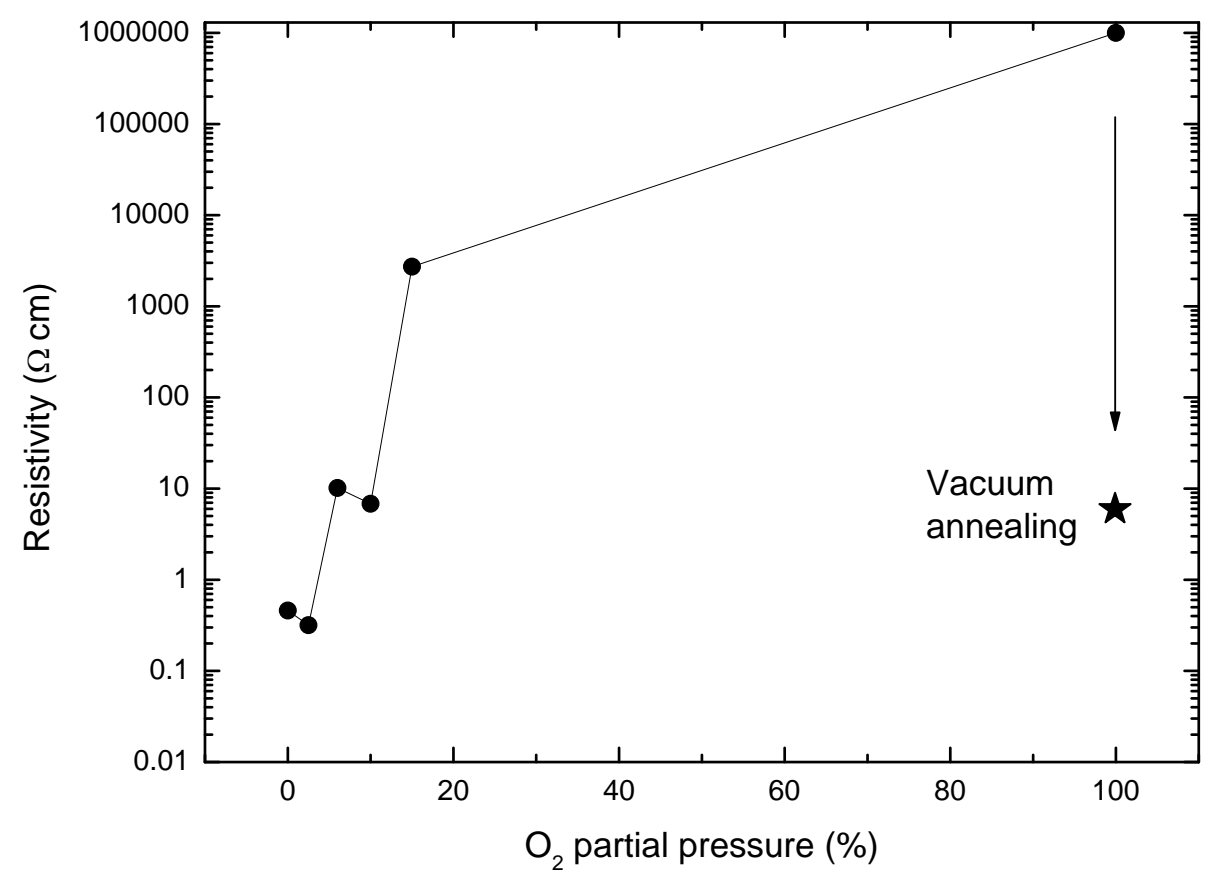

FIG. 5: In-plane electrical resistivity of hierarchical AZO layers as a function of oxygen partial pressure during deposition. The star represents a film annealed in vacuum.

There is an extremely significant increase in resistivity with increasing oxygen content, ranging from 0.3-0.4 $\Omega \mathrm{cm}$ for oxygen-deficient, conducting AZO $\left(\mathrm{O}_{2}\right.$ partial pressure < $5 \%$ ) to more than $10^{6} \Omega \mathrm{cm}$ for the case of almost insulating layers deposited in pure $\mathrm{O}_{2}$. Even though, in principle, it is not possible to exclude a small influence of morphology, the observed trend shows the importance of defects related to oxygen content in determining the electrical properties of AZO, whose resistivity spans across 7 orders of magnitude as a result of the variations in the $\mathrm{O}_{2}$ partial pressure during deposition.

To confirm the role of oxygen content, films deposited in pure oxygen were annealed in vacuum (i.e. a reducing environment) in order to induce $\mathrm{O}$ desorption. The effects can be seen in Fig.5 (point denoted by a star): the resistivity of the vacuum-annealed film reaches $6 \Omega \mathrm{cm}$. Since no significant morphology or crystalline domain size alterations appear upon annealing (not shown), this confirms the importance of oxygen content in determining electrical properties. On the contrary, an annealing treatment in air performed on oxygendeficient films (i.e. deposited in pure Ar) increases their resistivity over $1 \mathrm{M} \Omega \mathrm{cm}$, also in 
this case without significantly altering the morphology.

In addition, cross-plane electrical measurements were performed in the FIB-dual beam apparatus on selected $2 \mu \mathrm{m}$ thick AZO layers deposited on ITO substrates. Because of the porosity of the sample, the approach of the tip onto the AZO film was a critical step, therefore it was controlled under live SEM imaging in order to obtain similar conditions for each measurement. After lifting up the probe, a SEM image was taken to measure the tip contact area. As shown in Fig.6a, the AZO film is flattened under the tip pressure and the contact area is of the order of $20-30 \mu \mathrm{m}^{2}$.

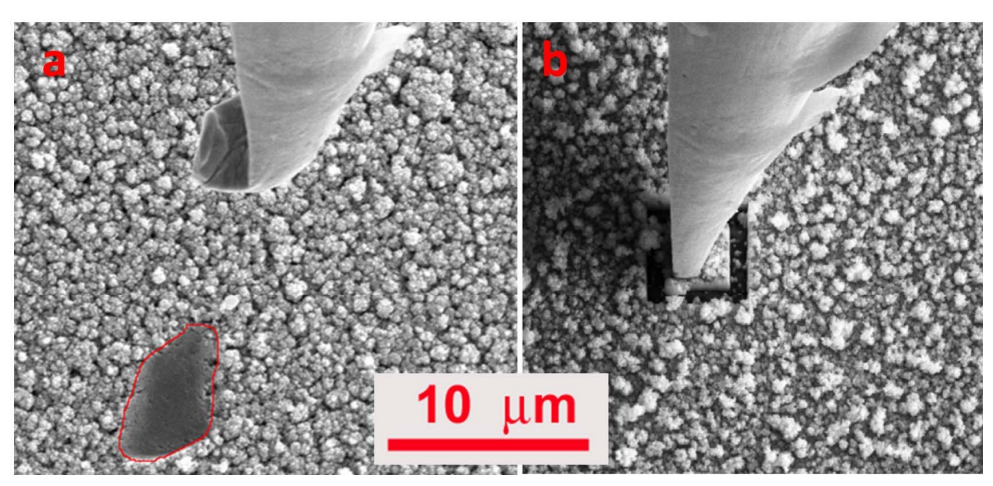

FIG. 6: a) SEM image of the tip contact area after an I-V measurement; b) SEM image of a tip contact in a square region delimited by a FIB-milled trench.

Our assumption is that conduction occurs mainly along the vertical column defined by the contact region (basis, measured from the trace left after each measurement) and the film thickness (height, of the order of $2 \mu \mathrm{m}$ for all the measured layers). To confirm this hypothesis we did the following experiment. We isolated a $5 \times 5 \mu \mathrm{m}$ region on the film by Focused Ion Beam-milling a square trench down to the ITO substrate (Fig.6b) and then compared the $\mathrm{I}-\mathrm{V}$ measurements obtained with the tip touching inside and outside the isolated region, respectively. The two curves had the same trend, current values being $25 \%$ lower for the measurement on the inside. Therefore we can conclude that $75 \%$ of the current measured on the bare film is flowing through the column under the tip contact area.

In Fig.7 the I-V curves measured over the $200 \mathrm{mV}$ bias cycle $(0 \mathrm{~V} \rightarrow+0.2 \mathrm{~V} \rightarrow-0.2 \mathrm{~V} \rightarrow 0$ $\mathrm{V}$ ) on three AZO films are shown. The maximum current decreases as oxygen concentration 

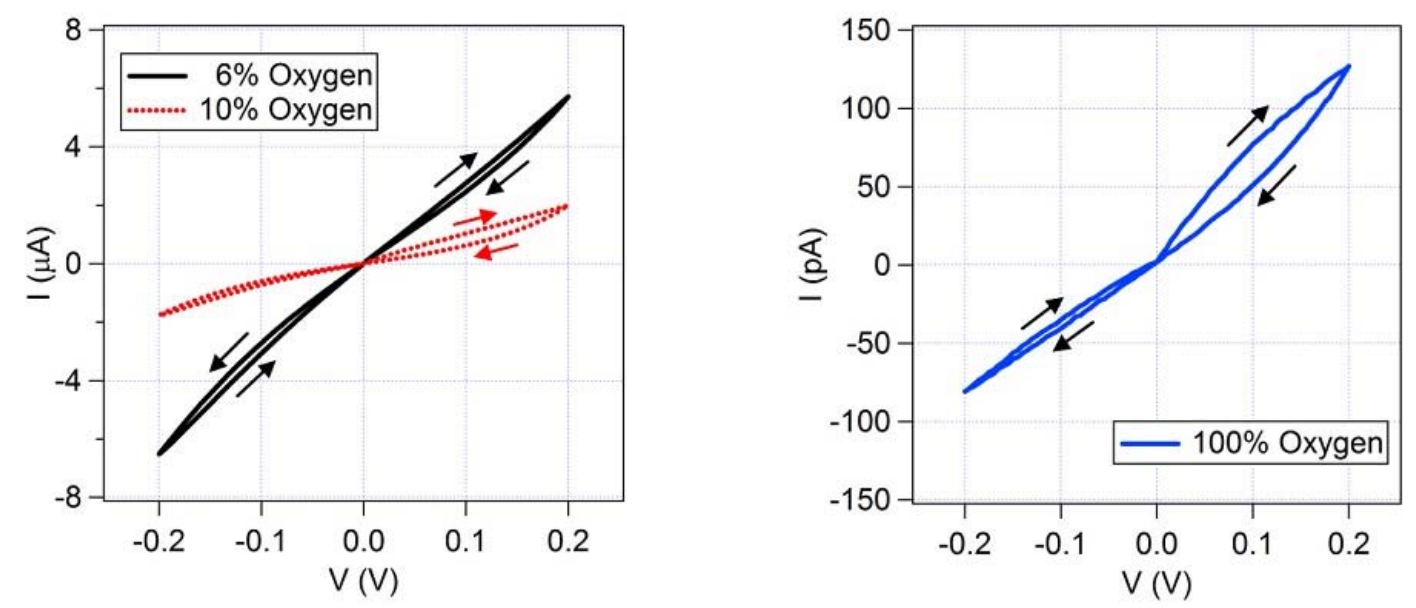

FIG. 7: I-V curves of hierarchical AZO layers grown at different Ar: $\mathrm{O}_{2}$ partial pressure ratios.

increases, the measured maximum values being $6 \mu \mathrm{A}, 2 \mu \mathrm{A}$ and $120 \mathrm{pA}$ for $6 \%, 10 \%$ and $100 \%$ oxygen content during deposition, respectively. All curves have a non-linear trend and show an hysteresis effect that increases with oxygen concentration, especially in the first part of the cycle. The non-linear trend can be due both to the porous structure of the film, which offers a random and discontinuous distribution of the conduction paths, and to a nonideal contact between the tip and the film that may introduce a Schottky-like barrier. The hysteresis recalls a capacitive (neighbouring trees charging up) or a memristor-like behavior, as shown by some metal oxides including compact AZO layers [28, 29] and solution-processed $\mathrm{ZnO}$ nanocrystals [30].

In table I, the resistivity values calculated considering the column under the tip contact and $75 \%$ of the measured current are reported. Cross-plane resistivity for the different deposition conditions appears to be larger than that measured along the in-plane direction, although in good agreement (same order of magnitude). This could be ascribed to the above mentioned more compact morphology at the film-substrate interface, considering that current is injected from contacts below the film in the case of in-plane measurements. 


\begin{tabular}{cccc}
$\mathrm{O}_{2}$ partial pressure & $6 \%$ & $10 \%$ & $100 \%$ \\
\hline$\rho(\Omega \mathrm{cm})$ & 50 & 240 & $3 \cdot 10^{6}$
\end{tabular}

Current flow area $\left(\mu \mathrm{m}^{2}\right) 24 \quad 30 \quad 22$

TABLE I: Estimated cross-plane resistivity of hierarchical AZO layers grown in different Ar: $\mathrm{O}_{2}$ atmospheres.

\section{CONCLUSIONS}

Hierarchically assembled forest-like AZO layers were grown by PLD at room temperature in a background atmosphere at a constant total pressure of $110 \mathrm{~Pa}$ and for different $\mathrm{Ar}: \mathrm{O}_{2}$ mixtures, in order to investigate the effects of stoichiometry/oxygen content in AZO while maintaining a mesoporous morphology, which is interesting in view of applications requiring transparent electrodes with strong light scattering and large surface area. The hierarchical nature of AZO emerged at higher $\mathrm{O}_{2}$ deposition pressures, and a moderately preferential growth direction along the vertical axis was found. The effects of $\mathrm{O}$ content on optical properties were investigated, identifying a significant decrease of the optical attenuation coefficient upon saturation of $\mathrm{O}$ vacancies, and very large haze values (i.e. light scattering power). Electrical properties were probed both in the in-plane and in the cross-plane direction, finding significant correlation with oxygen content in both cases, and conducting nanoporous layers can be realized by deposition in an oxygen-poor atmosphere. In view of the possible implementation of the tree-like architecture as a photoanode in solar devices, we could assess that the optimal combination of optical and electrical properties is represented by an oxygen-containing deposition atmosphere with oxygen content up to about $10 \%$. In perspective, a full understanding of the structure/property relation, and in particular of the different roles of morphology and defect chemistry, would be important for optimization of functional properties, opening the way for application in advanced device architectures.

This approach can allow to obtain large surface area nano- and mesoporous transparent scattering layers, with improved conductivity, of potential interest for transparent electrodes and photoanodes in organic and hybrid photovoltaic devices. 
[1] Tadatsugu Minami, Transparent conducting oxide semiconductors for transparent electrodes, Semiconductor science and technology, 20 (2005) S35-44.

[2] Ü Özgür, Ya I Alivov, C Liu, A Teke, M A Reshchikov, S DoÄßan, and V Avrutin, A comprehensive review of $\mathrm{ZnO}$ materials and devices, Journal of Applied Physics 98 (2005) $1-103$.

[3] Ho-Kyun Park, Jae-Wook Kang, Seok-In Na, Don-Yu Kim, and Han-Ki Kim, Characteristics of indium-free $\mathrm{GZO} / \mathrm{Ag} / \mathrm{GZO}$ and $\mathrm{AZO} / \mathrm{Ag} / \mathrm{AZO}$ multilayer electrode grown by dual target DC sputtering at room temperature for low-cost organic photovoltaics, Solar Energy Materials and Solar Cells 93 (2009) 1994-2002.

[4] B Rech, O Kluth, T Repmann, T Roschek, J Springer, J Müller, F Finger, H Stiebig, and $\mathrm{H}$ Wagner, New materials and deposition techniques for highly efficient silicon thin film solar cells, Solar Energy Materials and Solar Cells 74 (2002) 439-447.

[5] D Calestani, F Pattini, F Bissoli, E Gilioli, M Villani, and a Zappettini, Solution-free and catalyst-free synthesis of ZnO-based nanostructured TCOs by PED and vapor phase growth techniques, Nanotechnology 23 (2012) 194008.

[6] Z.L. Pei, X.B. Zhang, G.P. Zhang, J. Gong, C. Sun, R.F. Huang, and L.S. Wen, Transparent conductive $\mathrm{ZnO}: \mathrm{Al}$ thin films deposited on flexible substrates prepared by direct current magnetron sputtering, Thin Solid Films 497 (2006) 20-23.

[7] Kyung Ho Kim, Kazuomi Utashiro, Yoshio Abe, and Midori Kawamura, Structural Properties of Zinc Oxide Nanorods Grown on Al-Doped Zinc Oxide Seed Layer and Their Applications in Dye-Sensitized Solar Cells, Materials 7 (2014) 2522-2533.

[8] Jun-ichi Oda, Jun-ichi Nomoto, Toshihiro Miyata, and Tadatsugu Minami, Improvements of spatial resistivity distribution in transparent conducting $\mathrm{Al}$-doped $\mathrm{ZnO}$ thin films deposited by DC magnetron sputtering, Thin Solid Films 518 (2010) 2984-2987.

[9] M. Birkholz, B. Selle, F. Fenske, and W. Fuhs, Structure-function relationship between preferred orientation of crystallites and electrical resistivity in thin polycrystalline $\mathrm{ZnO}$ : Al films, Physical Review B 68 (2003) 205414.

[10] A V Singh and R M Mehra, Highly conductive and transparent aluminum-doped zinc oxide thin films prepared by pulsed laser deposition in oxygen ambient, Journal of Applied Physics 
90 (2001) 5661-5665.

[11] H Agura, Low resistivity transparent conducting Al-doped $\mathrm{ZnO}$ films prepared by pulsed laser deposition, Thin Solid Films 445 (2003) 263-267.

[12] Jurgen Lackner, Industrially-styled room-temperature pulsed laser deposition of $\mathrm{ZnO}: \mathrm{Al}$ films, Thin Solid Films 494 (2006) 302-306.

[13] Bin-Zhong Z Dong, Hao Hu, Guo-Jia J Fang, Xing-Zhong Z Zhao, Da-Yu Y Zheng, and YuanPing P Sun, Comprehensive investigation of structural, electrical, and optical properties for ZnO:Al films deposited at different substrate temperature and oxygen ambient, Journal of Applied Physics 103 (2008) 073711.

[14] P Gondoni, M Ghidelli, F Di Fonzo, M Carminati, V Russo, C E Bottani, A Li Bassi, and C S Casari, Structure-dependent optical and electrical transport properties of nanostructured Al-doped ZnO, Nanotechnology 23 (2012) 365706.

[15] P. Gondoni, P. Mazzolini, V. Russo, A. Petrozza, A. K. Srivastava, A. Li Bassi, and C. S. Casari, Enhancing light harvesting by hierarchical functionally graded transparent conducting Al-doped ZnO nano- and mesoarchitectures, Solar Energy Materials and Solar Cells 128 (2014) $248-253$.

[16] C.S. Casari and A. Li Bassi, Pulsed laser deposition of nanostructured oxides for emerging applications, In A. K. Srivastava, editor, Oxide Nanostructures: Growth, Microstructures and Properties, pages 99-114. Pan Stanford Publishing, 2013.

[17] Klaus Ellmer, Past achievements and future challenges in the development of optically transparent electrodes, Nature Photonics 6 (2012) 809-817.

[18] Anderson Janotti and Chris G. Van de Walle, Oxygen vacancies in ZnO, Applied Physics Letters 87 (2005) 122102.

[19] Anderson Janotti and Chris G. Van de Walle, Native point defects in ZnO, Physical Review B 76 (2007) 165202.

[20] Péter Ágoston and Karsten Albe, Intrinsic n-Type Behavior in Transparent Conducting Oxides: A Comparative Hybrid-Functional Study of $\mathrm{In}_{2} \mathrm{O}_{3}, \mathrm{SnO}_{2}$, and $\mathrm{ZnO}$, Physical Review Letters 103 (2009) 245501.

[21] P. Gondoni, M. Ghidelli, F. Di Fonzo, V. Russo, P. Bruno, J. Martí-Rujas, C.E. Bottani, A. Li Bassi, and C.S. Casari, Structural and functional properties of $\mathrm{Al}: \mathrm{ZnO}$ thin films grown by Pulsed Laser Deposition at room temperature, Thin Solid Films 520 (2012) 4707-4711. 
[22] M.K. Abyaneh, L. Gregoratti, M. Amati, M. Dalmiglio, and M. Kiskinova, e-J. Surf. Sci. Nanotech 9 (2011) 158.

[23] https://www.elettra.eu/elettra-beamlines/escamicroscopy.html.

[24] A. Bailini, F. Di Fonzo, M. Fusi, C. S. Casari, A. Li Bassi, V. Russo, A. Baserga, and C. E. Bottani, Pulsed laser deposition of tungsten and tungsten oxide thin films with tailored structure at the nano- and mesoscale, Applied Surface Science 253 (2007) 8130-8135.

[25] David B. Geohegan, Alex a. Puretzky, Gerd Duscher, and Stephen J. Pennycook, Time-resolved imaging of gas phase nanoparticle synthesis by laser ablation, Applied Physics Letters 72 (1998) $2987-2989$.

[26] Nurul Islam, T B Ghosh, K L Chopra, and H N Acharya, XPS and X-ray diffraction studies of aluminum-doped zinc oxide transparent conducting films, Thin Solid Films 280 (1996) 20-25.

[27] Wolfgang Körner and Christian Elsässer, First-principles density functional study of dopant elements at grain boundaries in ZnO, Physical Review B 81 (2010) 085324.

[28] Mandar M Shirolkar, Changshan Hao, Shiliu Yin, Ming Li, Haiqian Wang, Mandar M Shirolkar, Changshan Hao, Shiliu Yin, Ming Li, and Haiqian Wang, Influence of surface null potential on nonvolatile bistable resistive switching memory behavior of dilutely aluminum doped ZnO thin film, Applied Physics Letters 102 (2013) 243501.

[29] Shanshan Peng, Fei Zhuge, Xinxin Chen, Xiaojian Zhu, Benlin Hu, Liang Pan, and Bin Chen, Mechanism for resistive switching in an oxide-based electrochemical metallization memory, Applied Physics Letters, 100 (2012) 072101.

[30] J Wang, B Sun, F Gao, and N C Greenham, Memristive devices based on solution-processed ZnO nanocrystals, Phys. Status Solidi A, 207 (2010) 484-487. 